\title{
An observational study of children interacting with an augmented story book
}

\author{
Andreas Dünser ${ }^{1}$, Eva Hornecker ${ }^{2}$ \\ ${ }^{1}$ HIT Lab NZ, University of Canterbury, New Zealand \\ andreas.duenser@hitlabnz.org \\ ${ }^{2}$ Pervasive Interaction Lab, The Open University, Milton Keynes MK7 6AA, UK \\ eva@ehornecker.de
}

\begin{abstract}
We present findings of an observational study investigating how young children interact with augmented reality story books. Children aged between 6 and 7 read and interacted with one of two story books aimed at early literacy education. The books pages were augmented using animated virtual 3D characters, sound, and interactive tasks. Introducing novel media to young children requires system and story designers to consider not only technological issues but also questions arising from story design and the design of interactive sequences. We discuss findings of our study and implications regarding the implementation of augmented story books.
\end{abstract}

Keywords: Augmented reality, interactive book, storytelling, young readers

\section{Introduction}

Augmented reality (AR) technology has been used to develop different kinds of educational and entertainment applications. AR allows the user to experience the real world with computer generated content embedded into it. The user can not only view virtual content but also interact with it in real time.

An approach of augmenting a traditional medium with virtual content was realized with the MagicBook [3]. Various implementations of the MagicBook paradigm range from computerized "pop-up books" that allow the user to see animated 3D content and associated sound (e.g. the eyeMagic book [8]) and books that allow users to interact with the virtual content (e. g. the AR Volcano [12]), to books in which the user can seamlessly move inside the books' virtual content, being fully immersed in a virtual environment [3]. Augmented books have caught the attention not only of researchers but also of educators as a means to enhance books with interactive visualization and simulation, animation, 3D graphics and sound [11]. Such features added to a physical book can enhance the users learning experience by actively exploring and manipulating the medium. Using these tools and presentation methods can lead to a better understanding of complex dynamic processes or 3D structures and overcome limitations of conventional educational media. Besides the realization of a 
more adequate representation of specific types of content, interactive 3Dvisualizations may motivate learners and enhance engagement, supporting immersive learning $[4,8]$.

Instead of only displaying and interacting with book content on computers, a book created using AR technology enables the integration of tangible user interaction, which can support learning and collaboration between users [9, 10]. Users can navigate through the book by turning pages of a physical book. Integration of other interaction tools may allow users to further interact with certain elements and actively become part of the story.

Learning and comprehension can be supported by interaction, self directed learning, exploration and collaboration [6]. Loftin [5] argues that educators generally agree that experience is the best teacher. However, in reality students are seldom given the opportunity for direct experience of what is to be learned. Incorporating new media in education can augment the reading and learning experience [5].

With an interactive book there is a range of factors contributing to the user experience, from technological implementation to the integration and design of interactive parts. In this paper we will mainly focus on the latter.

\section{AR story books}

Augmented story books can be realized for expressive activity, for example letting children create their own stories and pictures by using AR-technology [8]. Some researchers also discuss different ways of non-linear story telling allowing the reader to pick different paths in a story or to change the plot according to the path taken.

The type of learning system analyzed here is targeted at what Marshall et al [7] call an 'exploratory activity' where a learner explores a model embodied in the system. Naturally the story itself determinates whether a book is engaging. While Mckenzie [5] identifies non-linear and interactive stories and activity books as options for ARbooks, the stories used in this study, while being interactive, followed a sequential story line. The stories allow the readers to interact with given story elements. The readers have to complete tasks but do not change the actual plot.

The BBC is running a project evaluating the use of AR books for early literacy in classrooms (children aged 5-7). In collaboration with this project, we use two of these AR books to study children reading and interacting with the books in single and pair settings outside of classrooms. Our aims were to explore how children interact with and handle the augmented books, how the integration of interactive story elements may enhance the reading and learning experience, and how the books support collaborative learning.

We have analyzed videos from eight pairs and six individuals, focusing on their collaborative behavior (pairs only), engagement with the story, and difficulties in interacting with the system. We will first describe the system and our study design. Then we will describe and discuss our findings focusing on issues regarding the design of AR based story books. 


\section{The observational study}

\subsection{The system}

The AR books were created using ARToolkit technology [2]. Physical pages (sheets of paper) contain markers and the stories' main characters are represented by paddles also with markers on them.

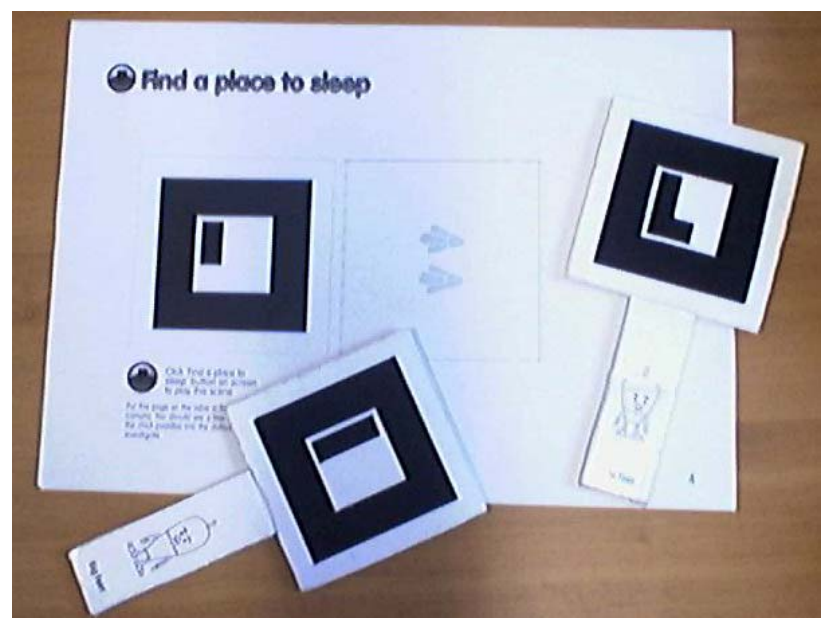

Fig. 1.: Page and paddles of the "Little Feet and Big Feet" AR story book

The story-packs include files to be printed out and be used as the books pages or to be cut into shape for paddles. We glued the pages and paddles on cardboard to make them more sturdy and stable. A web-cam is mounted on top of a computer screen. Once the markers are within camera view the augmented book content becomes visible on the screen (overlaid onto markers). This setup allows the user to see real and virtual content in a combined view in front of them as well as themselves interacting with the story (see Figure 3).

An advantage of this setup is that it can be used on standard computer equipment. Hence it can be used in most modern classrooms or homes without requiring expensive additional hardware. However, it does not provide an integrated view of real and virtual objects like other AR setups using see-through devices.

The computer screen shows text pages of the stories and navigation buttons for going to the next text page or back, skipping to another section of the book, and buttons to listen to the story and to close the window (the computer mouse is used for these elements).

The physical pages in combination with the paddles are used during the interactive sequences. The augmented book is distributed across screen and paper pages serving different purposes. 


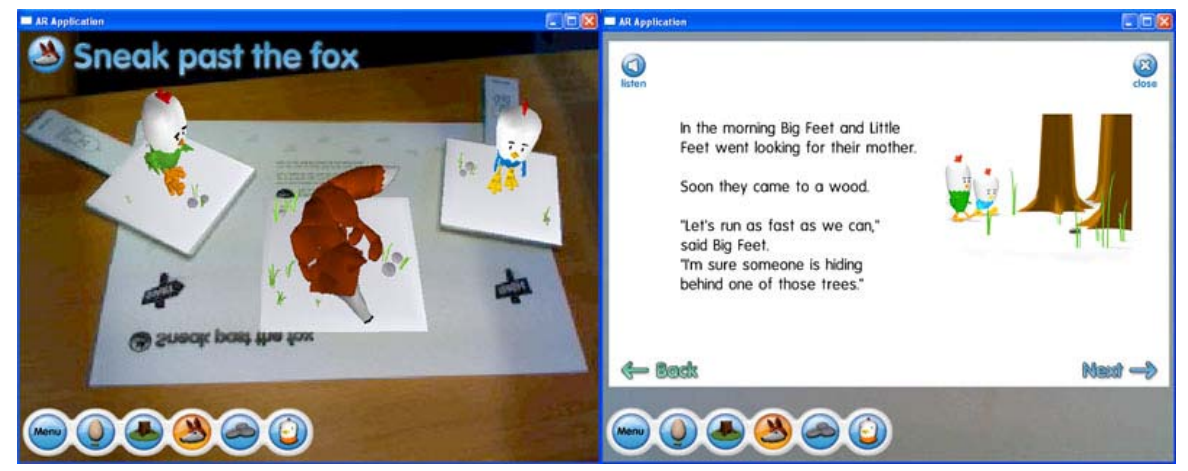

Fig. 2. Example for interactive screen (left) and text page (right)

\subsection{The story books}

We used two story-books. "Big Feet and Little Feet” (referred to as 'chick story') tells the story of two little chicken who have been left behind and have to overcome several obstacles to find their mummy. The other book "Looking for the sun" (referred to as 'sun story') has four insect characters (and thus four paddles) who try to find the sun. The chick story had been specifically written for the AR-Jam while the sun story was adapted from an existing book by a children's book author.

The stories start with text pages on the computer. The children can choose to read the stories or click on the listen button to have the story read to them by a prerecorded voice. When there are more text pages they click on a "Next" button. After each text section a short instruction tells the children to close the screen-window which leads to the interactive sessions. Here the children have to solve different scenarios and interact with content displayed on the book-pages and paddles. The pages usually have 'hot spots' next to the markers, indicated by a grey outline or other drawings. Placing paddles on a hot spot usually triggers certain events. In Fig. 2 the chicken have to sneak past the fox (following footprints from the start to the finish signs) without waking him up. When they are getting too close, the fox starts moving and growling.

After completing these sequences the readers have to mouse-click on "Next scene" to get to the next text page or to click on "Play again" to repeat the interactive sequence. As each interactive sequence is represented on separate book pages the children have to turn the (physical) pages before starting with a sequence. In the sun story one sequence stretches over 4 different pages requiring the children to flip pages during one interactive sequence.

Having two stories, we decided to employ both of them. This would allow a comparison and provide us with more insight into relevant design issues. Pilot study observations indicated that the sun story somehow lacked flow and engaged the children less. We included this story in the study, as we hoped from a comparison to better understand what exactly the problems were. 


\subsection{Study design and method}

Children aged $6 \frac{1}{2}$ to 7 from a local primary school participated in the study at a local library learning centre. For this study, avid and good readers were solicited. As we were interested in how interactivity supports collaborative learning, we decided to include pairs and individual readers.

Pairs were well acquainted with each other. From the pair condition we expected closer insight into the children's thoughts and opinions as constructive interaction may create a natural communicative situation alleviating the problems of think-aloud methods [1]. By observing individual readers we wanted to study how young children can read and interact with the stories and handle the technology when on their own.

Six pairs and six individual children 'read' and interacted with one of the two augmented books. During this phase two researchers were present and supported the children when they got stuck. Each story was read by three pairs and three individual readers. After completing the story, each child was interviewed individually following a semi-structured interview. As only the interview questions changed, we include the video data from our pilot study (two pairs) in our analysis. The children were videotaped with the written consent of their caregivers and the school. The videos have been analyzed by the authors in shared analysis sessions, taking extensive notes of children's actions, nonverbal behaviors, and talk. Analysis was open-ended, iteratively evolving and collecting instances of the issues we here report upon.

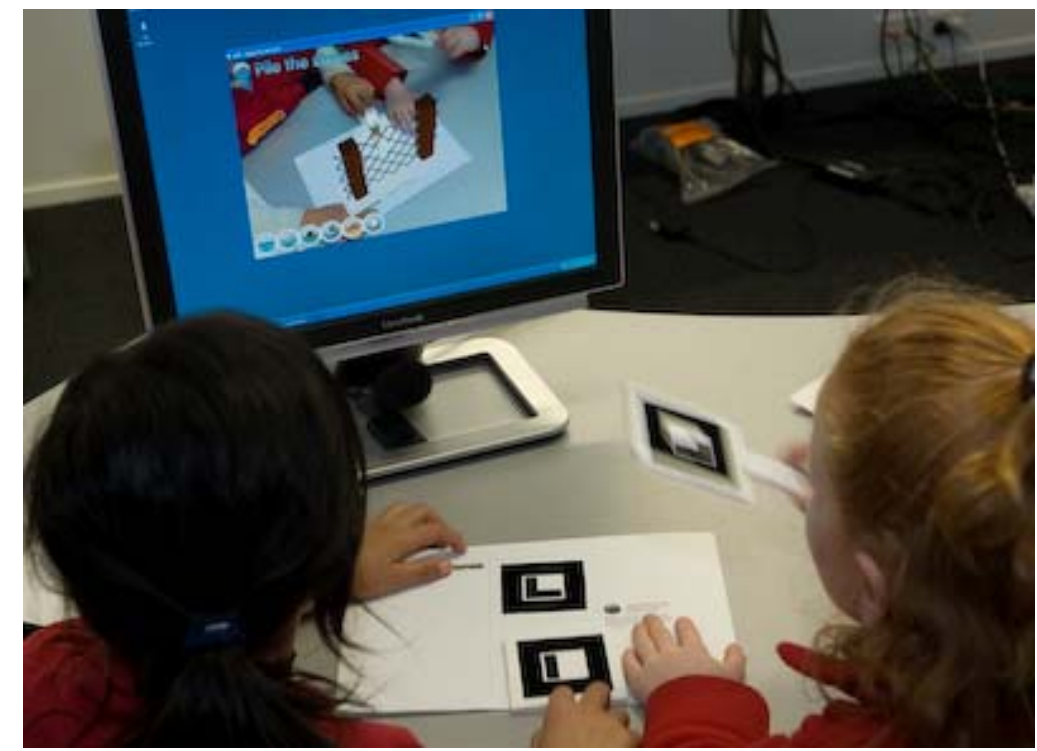

Fig. 3. Children interacting with the augmented book 


\section{Findings}

\subsection{General Findings and Observations}

The collaborative behaviors of the pairs were very diverse. We feel that the diversity we saw with only eight pairs indicates that a multitude of styles exist between peaceful collaboration and conflict or struggle over control, equal contribution and dominance, fluid roles or clear 'distribution of labor', and impulsiveness and shyness.

Interaction styles with the story and interactive sequences were quite diverse, with playful and experimental interaction (sometimes just for the fun of it) at one end of the spectrum, and problem-solving, strategic interaction at the other end. There were clear differences discernible as to whether children were acting playfully and exploring features of the AR book, or whether they were intent on executing the interactive sequences 'correctly' on the first try. As we will discuss later, this interaction style seems to be correlated with some of the phenomena and issues we uncovered.

We found that after having support for the first two sequences and having understood how to move from sequence to sequence, most children were able to interact with the system without much prompting. When the story has a clear structure and story line and the sequences clearly signal when to move on, we would expect the children to be able to read another book without much assistance. This observation is based on the findings of this study using a sample representing the upper end of a class (i.e. good readers). Early observations of our second study (which is not yet fully analyzed) indicates that this is to a certain extend also true for low ability readers.

\subsection{Design of interactive sequences}

With an AR-book, the contribution of the interactive sequences to the story is important. Classic elements of storytelling should also be considered, such as choice of main characters, setting, and story plot. We found that sequences that did not advance the story were not satisfying for our young readers and sometimes left them confused, particularly when there was no clear signal provided on whether or not the sequence had been successfully completed. This signal can lie in the scheme of the sequence or be explicitly provided.

With the chicken story the interactive sequences usually had the story characters overcome a problematic situation (classic story structure of anticipation and climax), with the chicks e.g. getting out of their eggs or climbing through a fence to escape a fox. In the example of climbing through the fence the two chickens walk off the screen. Children almost always reacted instantaneously to this prompt by ending the interactive sequence. In another instance where the interactive sequence does not solve the problem, the animated chicks say loudly "let's try somewhere else". Here some children correctly went on to the next scene, however, other children asked "but where should we try" and went on trying similar actions on the same page. 
In the sun story we observed more of this type of problems. This story has its characters try several things to reach the sun, however, they can't manage to reach it. This often leaves it somewhat unclear whether every possible approach had already been tried. We often saw children staring at the screen for several seconds, unsure if they should continue to try different things without noticing that they already have completed the sequence. Also, before starting with the interactive sequence some children said in advance that the characters can't reach their goal this way (e.g. reaching the sun by climbing on top of each other). With these sequences is doesn't seem very motivating to try to achieve the goal anyway.

Another problem we observed with the sun story was that the "Next scene" button was always available. Thus children could end the interactive sequences and move on to the next part without having finished the task. In the chicken story it only appeared once the sequence was completed and the goal was achieved. Appearance of the button thus provided an implicit signal for the children.

\subsubsection{Role of interactive sequences}

Interactive sequences in a story book can be used to retell / recreate or to advance the story plot. In the first case, the reader interacts with content that has already been told in the story. We observed that in such sequences some children tried to remember details and to do things correctly without experimenting or playing around too much. For example in the sun story the children first read which animal liked which food. In the following interactive sequence they were asked to find the right animal for the right food by putting a character next to the particular food displayed. Thus some children first tried to remember the correct order before even picking up a paddle and trying it out. When they achieved the goal they moved on without further exploring other possibilities.

When events of an interactive sequence were not already pre-primed, the children seemed to show more playful behavior as they had to discover what had to be achieved. However, it is important to give the children at least some instruction or hints on what they are expected to do.

Naturally, the decision of recreating or advancing the story depends on the story design and the didactic aim. However, advancing the story plot seems to lead to greater enjoyment and promote exploratory behavior. Children that were playfully discovering things were more likely to repeat a sequence for the fun of it (or to let both of a pair perform the events) and seemed to be more engrossed and enjoying themselves.

\subsubsection{Introducing interaction possibilities}

The first interactive sequence of a book should engage readers and ask them to explore how to interact with the system. Especially novice users should have a chance to get used to new kinds of interaction techniques. For example, in the first sequence of the chick story the children had to help the chicken to get out of the eggs. They had to bang the paddles (displaying eggs) together several times. By doing this the eggs cracked and the two chickens got out. This seemed to give the children a good idea on how to use the paddles and interact with the system. 
In the other story the first interactive sequence was rather static and children tended to transfer their experience to the next sequence and had to be scaffolded to try different manipulations; e.g. to place paddles next to each other or to move paddles around instead of removing a paddle from the working area when taking and using another one.

\subsubsection{Instructions for interactive sequences}

We found that it is very important that instructions should be clear, especially when the children are expected to experience the story without assistance. Sometimes the children seemed to be confused by instructions. In the first interactive sequence of the sun story the children were expected to move the paddles close together triggering the characters displayed on the paddles to wave. The instructions for this page are: "Can you tell what animals they are? Close this window and look at the 'Say hello' page." Some children then had a look at the paddles, without moving them around, told what animals they were (pictures of the animals are printed on the paddles; see Fig. 1) and moved on to the next page. In one instance a child, after being instructed to 'close this window' closed the application window (by clicking on the operating systems' close button) instead of closing the text window by using the dedicated close button (Fig. 2).

\subsection{Pairs and individual readers}

An interesting observation was that difficulties with story flow and discovery of how to do things were more pronounced when reading individually. Single children got stuck more often. Collaborative interaction seemed to help children to cope with problems more easily and increased the likelihood of the children trying alternative interactions. However, with some pairs we also observed the contrary. Some copied behavior from their partners which often resulted in both children doing the same things over and over again without having any progress. Also pairs, being more playful, seemed to be less strategic in their behavior.

The number of paddles used for interaction may have an influence on collaboration. We observed that the children liked to identify with a character. One child for example said: "I was Big Feet, however, my friend should have been Big Feet because he has bigger feet”. Identification might be easier if only two characters are used so that each child has one specific paddle / character. Additionally it seems to depend on the story-characters themselves (e.g. are they heroes) whether children identify with them. The sun story had four characters and thus four paddles. Although some children mostly used "their two paddles", identification with the characters was less obvious.

Interacting with the sun story took individual readers rather a long time. We observed that in this story, individual readers got stuck more often than pairs. Pairs, especially the playful ones, took more time for the chick story than singles. In this story the playful pairs repeated interactive sequences they liked quite often (e.g. cracking the eggs). Sometimes we even had to ask them to stop and to move on to the next part. In general, single readers seemed to be less playful and did not choose to 'play again' as often. When they repeated a sequence, it appeared that they had not 
understood what they had done, or did not notice that they had got it right. Longer interactive times for single children were rather related to frustration and problems than to playfulness.

Tab 1: Overall times and times for interactive sequences

\begin{tabular}{|l|c|c|c|c|}
\hline \multirow{2}{*}{} & \multicolumn{2}{|c|}{ overall time } & \multicolumn{2}{c|}{ interactive time } \\
\cline { 2 - 5 } & single & pairs & single & pairs \\
\hline chick & $0: 10: 46$ & $0: 17: 31$ & $0: 05: 49$ & $0: 09: 43$ \\
\hline sun & $0: 18: 12$ & $0: 16: 50$ & $0: 14: 09$ & $0: 11: 54$ \\
\hline
\end{tabular}

\subsection{What did you like best?}

After the children read and interacted with the story we interviewed them following a semi-structured interview. We asked the children what they liked best. Most mentioned events from the interactive sequences. From the chick story, cracking the eggs was mentioned four times, the 'going through the fence' sequence three times, and sneaking past the fox twice. One child liked best that the chickens made their way home.

Children who read the sun story liked the catapult activity best (four times) and one child liked building the tower. Other elements that were mentioned for the sun story were more related to events in the story than to actual activities. The part when Scuttle, who landed on a dog's head, told the others that the sun was smelly was mentioned twice. Two children liked the character Claws and that he suggested different things (e.g. "Let's go and sunbathe”).

There were also answers concerning interaction or interactive sequences in general. One child said: "I liked how we had to get it right; the eating thing; I liked how we had to sort of figure out how" and another one "I liked the activities but they were quite hard, to know where to put the things". While holding and moving the paddles during the interview one child told that he liked best "using these things" and that "they moved" (the characters).

Another question we asked was how they would describe the activity to their siblings or best friend. Answering this question seemed a little bit harder for the children. Some didn't know how they could describe their experiences. Most answers were related to the story or reading. Five children said that they read a story or read a story on a computer. Two said that they listened to a story. Others would explain the story, that it was a fairytale, that it was funny, talk about the characters or just say what they remembered. Several of the children's answers also included interaction elements: "We held the chickens with these things, and we made them go upside down 'bang' [with hand gesture]”, “you got to like control Little Feet and Big Feet; you got to do the things with the characters", "I can describe how they moved", "I did some things to work out the things, to make things happen."

We also asked children who only described story events to try to explain how they read the story. Answers ranged from using a computer and watching to "moving these sort of things" (referring to the paddles). 


\section{Conclusion}

Our analysis revealed some interesting observations regarding the design of augmented reality story telling books in which children "read" a predefined story with a linear story plot. However, some findings may also be relevant for other kinds of augmented books, for example including active storytelling by children who develop their own story.

We found that the story and interaction sequences should be appropriate and demanding enough for the users, in our case for young children. The story should be engaging and include a clear structure and climax. In general, the integration of interactive sequences using AR technology should augment and illustrate the text and contribute in a satisfactory way to the story (e.g. advance a plot).

When children are meant to experience the story on their own, book creators should make sure that concepts integrated are well understood by all children (e.g. some children did not know what a catapult was), especially in interactive sequences. There should be clear signals if the goal of an interactive sequence is achieved and as to when the children should move on to the next parts.

Individual readers seemed to have more difficulties with story flow and discovery of how to accomplish the various tasks. Single readers often showed more strategic behavior and seemed to be less playful.

When children read and interacted with the stories in pairs they could help each other out in situations where individuals often got stuck. Collaboration also can increase chances of finding alternatives when certain actions did not support task completion. On the other hand, some children just copied behavior of their partners and vice versa. These pairs often got stuck with certain tasks and kept doing the same thing over and over again.

Most children mentioned events of interactive sequences when we asked them what they liked best. Although the children had difficulties verbalizing their experiences, a lot of them also referred to the system and augmented book itself. They especially liked to move and interact with the paddles and characters. Letting children interact with the story seems not only to be quite engaging but also might facilitate recall of story events.

With data from our second study involving low ability readers we hope to get a better understanding of which readers can benefit most from this kind of literacy learning medium and how to design interactive AR books to be suitable for a broad range of readers. 
Acknowledgements. We thank Christchurch South Learning Center, Nina Whitfield, Thorrington School, all participants and their parents, the BBC, Brendan Lancaster (content producer), Adrian Woolard, and Hilary Smith for discussions. This study was financed via the MagicBook grant (NERF UOCX0204).

\section{References}

1. Als, B., Jensen, J., Skov, M.: Comparision of Think-Aloud and Constructive Interaction in Usability Testing with Children. IDC 2005. ACM Press (2005) 9-16

2. Billinghurst, M., Kato, H.: Real World Teleconferencing. ACM CHI'99, Pittsburgh, PA (1999) 194-195

3. Billinghurst, M., Kato, H., Poupyrev, I.: The MagicBook - Moving seamlessly between reality and virtuality. IEEE Computer Graphics and Applications 21 (2001) 6-8

4. Dünser, A., Kaufmann, H., Steinbügl, K., Glück, J.: Virtual and Augmented Reality as Spatial Ability Training Tools. CHINZ 2006. ACM Digital Library, University of Canterbury, Christchurch, New Zealand (2006) 125-132

5. Loftin, B., Engelberg, M., Benedetti, R.: Applying virtual reality in education: A prototypical virtual physics laboratory. Proceedings of the IEEE 1993 Symposium on Research Frontiers in Virtual Reality. IEEE Computer Society Press, Los Alamitos, CA (1993) 67-74

6. Mantovani, F.: VR Learning: Potential and Challenges for the Use of 3D Environments in Education and Training. In: Riva, G., Galimberti, C. (eds.): Towards CyberPsychology: Mind, Cognitions and Society in the Internet Age. IOS Press, Amsterdam (2001) 207-225

7. Marshall, P., Price, S., Rogers, Y.: IDC ’03. ACM Press (2003) 101-109

8. McKenzie, J., Darnell, D.: The eyeMagic Book. A Report into Augmented Reality Storytelling in the Context of a children's workshop 2003. New Zealand Centre for Children's Literature and Christchurch College of Education (2004)

9. O’Malley, C., Fraser, D.S.: Literature Review in Learning with Tangible Technologies. NESTA Futurelab Report 12 (2005)

10.Price, S., Rogers, Y., Scaife, M., Stanton, D., Neale, H.: Using 'tangibles' to promote novel forms of playful learning. Interacting with Computers 15 (2003) 169-185

11.Shelton, B.: Augmented Reality and Education. New Horizons for Learning. http://www.newhorizons.org/strategies/technology/shelton.htm (2002)

12.Woods, E., Billinghurst, M., Looser, J., Aldridge, G., Brown, D., Garrie, B., Nelles, C.: Augmenting the Science Centre and Museum Experience. 2nd international conference on Computer graphics and interactive techniques in Australasia and SouthEast Asia (Graphite 2004). ACM Press, Singapore (2004) 230 - 236 